IIUC STUDIES

ISSN 1813-7733

Vol.-12 December 2015

(P. 41-62)

\title{
Chittagonian Variety: Dialect, Language, or Semi-Language?
}

\author{
Muhammad Azizul Hoque ${ }^{1}$
}

\begin{abstract}
The main concern of this paper is to explore the status of Chittagonian variety- a language spoken by the people of Chittagong in Bangladesh-with a view to considering whether it is a dialect of Bangla or a language or a semi-language. Though it is closely related to Bangla, its distinctive features like a different sound system, mutual unintelligibility with Bangla, etc. give it a separate entity and encourage the researcher of this paper to rethink about the existing idea of its being a dialect of Bangla and to try to present some supportive argumants in favor of its separate entity as a language. In the article the researcher has provided an introduction to the Chittagonian variety; language and dialect; previous literature, and used methodology consisting of random sampling based on the collection of newspaper articles, books, and internet sources, and the like. And some findings from that research and recommendations have been discussed.
\end{abstract}

\subsection{Introduction}

The history of sociolinguistics reveals that some languages like Sanskrit and Latin are dead for the lack of proper maintenance and some others like English, French, and Italian have got the status of standard languages. Likewise, the spoken language of Chittagong Chittagonian has also got such characteristics as to be a distinct language. So it is not difficult for Chatgaya to be developed into a full-fledged language. But the Chittagonian variety, as the researcher observes, is losing its speakers day by day. Because thinking it as the substandard and distorted form of Bangla, the modern Chittagonian parents and guardians are encouraging their children to avoid using Chittagonian variety and to speak standard Bangla and English in their every day conversation. So it may be extinct in the very near future from the world history. Consequently, the world will lose not only a variety, but also its culture, literature and its overall heritage. Taking the matter into consideration seriously, the researcher concentrates on the Chittagonian

\footnotetext{
1 Assistant Professor, Department of English Language \& Literature, International Islamic University Chittagong. Phone: +8801818146370, E-mail: mazizhoque@yahoo.com
} 
variety regarding its status: whether it is a 'language', or a 'dialect' or more than a dialect.

\subsection{Review of Literature}

So far our study has investigated, the status of Chittagonian regarding its position is still an unexplored area in Bangladesh. But some studies maintaining the status of Chittagonian strongly support our area of study. Of these, the studies by Ahmad (2013), Wikipedia (2010) hold the view that Chittagonian is not a dialect of Bangla, rather it is a distinct language. Alam's (2012) observation too is supportive for claiming Chittagonian as a separate language. Alam, M. U. (2011) is confused if Chittagonian is a dialect or a distinct language. Moniruzzaman (2007) has studied Chittagonian rigorously, but he doesn't think that Chittagonian is a separate language. The studies by Khan (2009), Wasif (2012), Morshed (2007), and Sismhu (2011) are worth supportive for the status of Chittagonian. The study of McArthur (1998) regarding the status of Scots is similar to the nature of the present study and has encouraged us to frame our research.

The details of these studies are summed up in the section "Previous Literature and Chittagonian”.

\subsection{Language and Dialect}

According to Longman Dictionary of Language Teaching and Applied Linguistics;

A dialect is a variety of a language, spoken in one part of a country, by people belonging to a particular social class, which is different in some words, grammar, and/ or pronunciation from other forms of the same language. A dialect is often associated with a particular accent. Sometimes a dialect gains status and becomes a standard variety of a country. (Richard, et al, 1993, p. 107)

The distinction between them is quite ambiguous. Referring to Haugen (1966a), Wardhaugh (2006, P.28) has stated that for ordinary people a dialect is certainly no more than a local non-prestigious (therefore powerless) variety of a real language. In contrast, scholars often experience considerable difficulty in deciding whether one term should be used rather than the other in a certain situation.

Here what Spolsky (1998, p. 30) observes is noteworthy. He says that what language a dialect belongs to depends on social and political rather than purely linguistic factors. For example, he observes that SerboCroatian was considered by linguists as a language with regional 
variation until Yoguslavia was united. But 'with the separation into distinct and warring territories, and with the subsequent transfer of populations and insistence on ethnic difference, Serbian and Croatian have now emerged as distinct languages'. Contrastingly, in the Netherlands, 'only Frisian is recognized as a distinct language; all other regional varieties are labelled dialects. As a remark, he adds, 'a language is a dialect with a flag, or even better, with an army.'

Similarly, regarding distinction between 'dialect' and 'language' Crystal (2003, p.136) has stated that dialects are subdivisions of languages. It is usually said that people speak different languages when they do not understand each other. But so-called dialects of Chinese (Mandarin, Cantonese, etc.) are mutually unintelligible in their spoken form, though they share the same written language, which is the main reason to regard them as dialects of Chinese. And opposite situation occurs: Swedes, Norwegians, and Danes are generally able to understand each other, but their separate histories, cultures, literatures and political structures suggest them as different languages.

So considering the above statements about language and dialect, it can be said that both terms are ambiguous. From the definition of Longman Dictionary, we know that a dialect is different only in some words, grammar, and pronunciation. Chittagonian variety, we know, is vastly different in words and pronunciation from Bangla, though there is no noticeable difference in grammar. So let us discuss the Chittagonian Variety.

\subsection{Introduction to Chittagonian Variety}

Chittagong is the chief Indian Ocean port city in the south-east of Bangladesh. The name also refers to one of the six divisions in Bangladesh. It was formerly divided into two administrative regions north Chittagong and south Chitttagong. Cox's Bazaar was separated in 1954. From the geographical description and demographic profile, it is understood that Chittagong is an area which consists of the characters of several sub-islands and people of different cultures. The Buddhist culture of Arakan, the Muslims who migrated to Chittagong in the Mughal and Pre-Mughal, Portuguese period, etc. and the European culture, invading and introducing animistic religious culture of the aborigines in the Chittagong Hill Tracts, the mixed culture of Arakan, Burmese and Chakma-Burmese culture and lastly the modern Christian culture of the British occupation had made a great impact on this area. (Mniruzzaman, 2007) 


\subsubsection{Sounds /Phonological Description of Chittagonian}

From Moniruzzaman's study the following Sounds /Phonological Description of Chittagonian variety can be summed up:

\section{Vowels}

Seven vowels can be identified. They are: front, mid and back or, unround 3 , neutral 1 , and round 4 . The three front vowels are [i, e, æ]. The three back vowels are $[u$, o] (including an irregular vowel $[\varepsilon]$ which is lower than [æ] and higher than [e]). The only mid vowel is [a]. From his study it is known that almost all the vowels of Chittagonian can be nasalized. (Mniruzzaman, 2007)

From Wikipedia it is also known that nasalization of vowels is contrastive in Chittagonian, as with other Eastern Indic languages. A word can change its meaning solely by changing an oral vowel into a nasal vowel, as in আর ar "and" vs. আঁর ãr "my". Below are examples of Chittagonian phrases that include nasal vowels.

How are you: (Standard Bengali: তুমি কেমন আছ?): -তুঁই কেন আছো? Tũi ken aso?

I am fine: (Standard Bengali: আমি ভাল আছি।): -আঁই গোম আছি। Ãi gom asi. Where are you?: (Standard Bengali: তুমি কোথায়?): -তুঁই হোন্দে? Tũi honde?

In Chittagonian 'Tii' with nasalization is formal showing distant relationship, whereas in both Chittagonian and Bangla 'Tui' without nasalization is used for near and dear ones as well as inferior ones.

What's your name?: (Standard Bengali: তোমার নাম কী?): -তোঁয়ার নাম কী? Tõar nam ki?

My name is Nayan: (Standard Bengali: আমার নাম নয়ন।): -আঁর নাম নয়ন। Ãr nam Nayan.

I miss you: (Standard Bengali: তোমার অভাব অনুভব করছি): -ণোঁয়ার লাই আঁর ফেড ফুরের। Tõar lai ãr fed furer.

Where are you going? : (Standard Bengali: তুমি কোথায় যাচ্ছ?): --তুঁই হোন্দে যোর? Tũi honde jor?

Where are you from? :(Standard Bengali: তুমি কোথা থেকে আসছ?): - Tũi hothtun aishshu?

Where do you live? :(Standard Bengali: তুমি কোথায় থাক?): -- Tũi honde thako?

I live in Sultan Master Bari, Bengura, Boalkhali (Standard Bengali: আমি সুলতান মাস্টার বাড়ী, বেগুরুরা, বোয়ালখালি তে থাকি।): - Ãai Sultan Master Bari, Bengura, Boalkhalith thaki. 
I'm Iftekhar, grandson of Sultan Master (Standard Bengali: आমি ইফতেখার,সুলতান মাস্টার এর নাতি I): - Ãai Iftekhar, Sultan Masteror nathi. (Wikipedia, 2010)

Moniruzzaman's (2007) observation shows that in standard Bangla form there is no difference between long and short vowels. But in the Chittagong dialect, the reality is something different. These differences are found in-

Generally open or monosyllabic words;

1. In free variation, as : muchi/ muchi: (fly)

In the phonological environment

(phoneme) where the long vowel is essential, as:

roi (Sunday) / ro:i (to stay)/ kon (who, pronoun)/ ko:n (say, verb)

lor (to run)/ lo:r (take, verb, 2nd person)

\section{Compound Vowels}

Moniruzzaman's (2007) study also shows that various compoundings are formed when two or more vowels or vowel-like sounds are joined together. There are almost 33 diphthongs (13 with front vowe l+6 low vowe $1+14$ with back vowel). Again there are almost 12 triphthongs and 2 tetraphthorgs.

\section{Consonants}

He also observes that in terms of manner of articulation, the consonants of the Chittagong dialect are: plosive 19 (including 11 regular plus 8 irregular and peripheral), affricate or almost affricate 1 , spirant 6 (including regional variety and free variation), fricative 3 (including contrastive distribution of [s] and [S]), rolling 1, flap 1 and lateral 1.

a) Plosives: $\mathrm{P}^{/} \mathrm{P}^{\mathrm{h}}, \mathrm{b} / \mathrm{b}^{\mathrm{h}}, \mathrm{t} / \mathrm{t}^{\mathrm{h}}, \mathrm{d} / \mathrm{d}_{\mathrm{n}}^{\mathrm{h}}, \mathrm{t} / \mathrm{t}^{\mathrm{h}}, \mathrm{d} / \mathrm{d}^{\mathrm{h}}, \mathrm{J} / \mathrm{J}^{\mathrm{h}}, \mathrm{k} / \mathrm{k}^{\mathrm{h}}, \mathrm{g} / \mathrm{g}^{\mathrm{h}}$

b) Nasals: $m, n, q$

c) Affricates: / $\mathrm{t} \widehat{\mathrm{s}} /$ (phonological and orthographic representation [s]).

/ $\mathrm{t} \widehat{\mathrm{s}} /$ used in word-initial in open syllable and other position in close syllable, as -

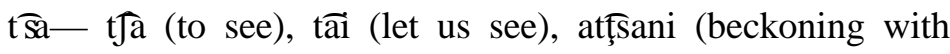
hand)

d) Fricatives: Moniruzzaman has identified the following fricative sounds in Chittagonian variety: $\phi, \quad f, \quad q \quad x, \quad y, \quad z$ (Moniruzzaman, 2007)

Chittagonian as mentioned in Wikipedia is distinct from Bangla because of its large inventory of fricatives, which often correspond with stops in 
Bangla. For example, the Chittagonian voiceless velar fricative [x] (like the Arabic "kh" or German "ch") in [xabar] corresponds to the Bangla voiceless aspirated velar stop [ $\left.\mathrm{k}^{\mathrm{h}}\right]$, and the Chittagonian voiceless labiodental fricative [f] corresponds to the Bangla voiceless aspirated bilabial stop $\left[\mathrm{p}^{\natural}\right]$. Some of these pronunciations are used in eastern dialects of Bangla as well. (Wikipedia, 2010)

\subsubsection{Grammar}

Chittagonian grammar is similar to that of Bangla, with significant variations in inflectional morphology (prefixes, suffixes, particles, etc.), and some variation in word order.

Like related languages of the eastern subcontinent, Chittagonian is a head-final language, with Subject-Object-Verb basic word order. Like Assamese (Ôxômiya) but unlike Bengali (Bangla), Chittagonian has preverbal negation. This means that the negative particle will precede the verb in Chittagonian, where the corresponding Bangla version would have a negative particle following the verb. (Wikipedia, 2010). In “বঙ্গবাণী”(Bongobani) by the famous Bengali poet Abdul Hakim, the use of preverbal negation is found:

যে সবে বঙ্গেত জন্মি হিংসে বঈবাণী। সে সব কাহার জন্ম নির্ণয় ন জানি।।

\subsubsection{Vocabulary}

Chittagonian has its own rich vocabulary. As found in Wikipedia (2010), Chittagonian includes a significant number of imported words from Arabic, Persian, and Turkish, as well as, to a lesser extent, Portuguese. In addition, English words are widely used in spoken Chittagonian. The contribution of Arabic, Persian, and Turkish words to Chittagonian Bengali is far greater than that to the standard. This is due to the fact that Chittagong was a port city that had been open to traders from Arabia, Persia and Turkey since ancient times, causing Chittagonian variety to absorb their words. Among Europeans, the Portuguese colonists were amongst the first to reach Bengal, and Chittagong as a port city, was for a time under the administration of the Portuguese. Consequently there are a larger proportion of Portuguese loanwords in the usage of Chittagonian speakers than that of standard Bengali speakers.

From the study of BSC (2011) it is known that Chittagonian has many words of its own which are not found in Bangla. Moreover, many foreign loan words that are found in Chittagonian are not found in Bangla. For these special features of Chittagonian, there is no scope to opine that Chittagonian is the distorted form of Bangla. Here is the list of some foreign loan words: 
i) thuder (ঠাডার)-thunder - (English)

ii) kisim-kisim (কিসিম কিসিম) - different kinds -(Urdu)

iii) Hot titi (इট্টিটি পাখি) - Hoten tit bird - (English)

iv) jozja (জবা) - over excitement - (Urdu)

v) dabai (দাবাই) - ('medicine' from Arabic dawa)

vi) doboz (দবজ) - strong- (Persian)

vii) chonna (ছন্না)-(changed from Persian),e.g. (জিন্ত ছন্না ধরা/ 'pretending to be a Jinn'—a spirit)

viii) bain duar (বাইন দুয়ার) - back door - (Persian)

ix) chiz (ठীজ) - thing - (Urdu). It is found in the Muslim dialects of other regions too.

x) mesal (মেসাল) -example - (Urdu). It is also found in the Muslim dialects of other regions.

xi) liccor (লিচ্চর) - shameless - (Urdu)

xii) chodok (ছড়ক্) -light - (origin was not found)

xiii) égana (এগানা) - relative - (Urdu)

xiv) Bégana (বেগানা) - not related - (Urdu). It is also present in the Muslim dialect of Narail.

xv) pil (পिল) - (English)

xvi) boyar (বয়ার) -wind - (Persian)

xvii) Gom (গম) -well - (Urdu)

xviii) bala (বালা) - good - (Persian). It is not the corrupt form of Bangla 'bhala' (good).

xix) poa (পোয়া) - boy - (from Dravidian 'pilla')

xx) gara (গারা) - danger/hole - (Arabic)

xxi) kuizza (কুইজ্যা) - a pile of straw - (Arabic)

xxii) lédo লেডঅ - (from English 'lay down')

xxiii) ãi (আই) - (from English 'I'). it is not the corrupt form of Bangla ‘आमि' (ami)

xxiv) boza (বজा) - egg - (Arabic). It is also present in the Muslim dialect of Narail.

xxv) gob (গব) - cock and bull story - (Hindi)

xxvi) vinsa (ভিংসা) - those who make fun- (Hindi)

xxvii) ugwá,duwá (উগ্যা, দুয়্যা) -one, two - (Portuguese)

xxviii) sodor bodor (চদর বদর) -disorder-(Arkani)

xxix) moskari (মস্কারি) - fun - (Urdu). It is found in the dialects of other regions of Bangladesh.

From the study of Kashem (2011) some words and grammatical specimen of Charyapada are exactly found in present Chittagonian language. For example, sion-চীঅণ—thin, duarot-দুআরত—by door (charya 
no. 3); kacchi-কাছি —'rope’ (charya no.7); pithot-পীঠত--at back (অধিকরণে '-ত' বিভক্তি) and the use of negative marker before verb (যেমন- ধরণ ন জাই) etc. are worth mentioning. (17 June 2011, The Daily Azadi)

The researcher identifies some words of Chittagonian the alternatives of which are not found in Bangla. For example, ডামিশ (damish)-'not clever', owl(from Arabic 'awl' meaning 'disorder'). The hesitation device 'geya' originated from Persian 'geyah' has no alternative in Bangla. The following verses of Sukumar Barua (সুকুমার বড়য়া) are notable:

দেয়ানা হাডর দেয়ানা/মস্ত বড় সেয়ানা,/গরু বেটের বাড়ি কিনের/এই টেঁয়া সেই টেঁয়ানা?/বেডার মত বেডা হইয়ে/তোরার মত গেয়া-না? (Hasan, 2010)

It has some words of common gender, i.e. 'Kura' (both cock \& hen). The alternative of it is not found in Bangla. But in the dialect of Narail, there is a similar expression 'kudo' seems to have derived from 'kunkro' (কুঁকড়ো) < Skt. 'kukkut' (কুক্ৰুট).

\subsubsection{Number Marking Articles}

Chittagonian has singular and plural marking articles. When they are used before nouns, they are indefinite, but when they are used after nouns, they are definite. án and wá mark the singular numbers and ğín and gún, mark the plural numbers.

\begin{tabular}{|l|l|}
\hline \multicolumn{1}{|c|}{ Singular } & \multicolumn{1}{c|}{ Plural } \\
\hline Fothú án (the picture) & Fothú Ğín(the pictures) \\
\hline Fata wá(the leaf) & Fata Ğín(the leaves) \\
\hline Tar gán(the wire) & Tar Ğin(the wires) \\
\hline Faár gwá(the mountain) & Faár gún(the mountains) \\
\hline Debal lán(the wall) & Debal lún(the walls) \\
\hline Kitap pwá(the book) & Kitap pún(the books) \\
\hline Manúish cwá(the man) & Manúish shún(the men) \\
\hline Uggwá fata (a leaf) & Hodún fata(some leaves) \\
\hline Ekkán fothú(apicture) & Hodigin Fothú (some pictures) \\
\hline
\end{tabular}

Sometimes indefinite singular markers are used after nouns as well. In that case, they are preceded by 'ugg' and 'ekk' meaning 'one/a', but indefinite plural markers are used as 'ún' and 'ien' preceded by 'hod' meaning 'some', when they are used after nouns.

\begin{tabular}{|l|l|}
\hline \multicolumn{1}{|c|}{ Singular } & \multicolumn{1}{c|}{ Plural } \\
\hline Fata uggwá(a leaf) & Fata hodún(some leaves) \\
\hline Fothú ekkán(a picture) & Fothú hodien(some pictures) \\
\hline Tar gán (the wire) & Tar Ğin (the wires) \\
\hline Duar gán (the door) & Duar gin (the doors) \\
\hline
\end{tabular}

(Chittagonian language, 2011) 


\subsubsection{Literature}

Chittagonian is rich in both oral and written literature. Though many works are not found in Chittagonian variety, the poets and literary figures of Chittagong background used many words of Chittagonian in their work of Bangla literature. Among them, Daulat Kazi, Alaol, Daulat Uzir, Bahram Khan, and Sabirid Khan are worth mentioning. From them the following words have been summed up:

1. pus (to ask)

ওক সম্বোধিয়া নৃপ করিল পুছার

(পদ্বাবতী-Padmabati by Alaol, edited by Syed Ali Ahsan, p.65)

2. chapai (hiding)

পরম যতনে শ্ক রাখিল ছাপাই। (as above)

3. baza (to confine/to detain)

আহার দেখিয়া যেন পক্ষীমনে বস। পশ্চাতে বাঝিলে ফাঁন্দে বড়ই কর্কস।(as above)

4. khate (to close eyes)

তা দেখিয়া লোকসবে চক্ষু খাটে ভএ। (হানিফার দিগ্বিজয় - Hanifa’s Victory of Direction by Sabrid Khan)

In Sabrid Khan's poetry words like sore-অওরে (meaning 'concealment'), kone-কনে (meaning 'who'), khesi- খেঁচি (meaning 'with force') etc. are also noticeable. (Kashem, 2011)

As the Chittagonian variety does not have any writing symbol, Chittagonian poets and literary figures have had tendency to contribute to literature using Bangla letters. Here are some examples from BSC's (2011) study:

प) গুপ্ত কথা কইশুন

একে একে সব

বানাউটি ন হয় কথা

ন অয় ইবা গব্।
ए) এত বড় ভিংছা জাতি বসত রাজ্য শেষ, মাইনসে মানুষ বেচি খায় এক আচানক দেশ ॥|

Local songs occupy a great part in Chittagonian literature. Among the Chittagonian local singers, the names of Shefali Ghosh, Kolyani Ghosh, and Shyam Shundor Baishnab lead the list. Here is a specimen of the local songs of Kolyani Ghosh (1998, p. 374): 


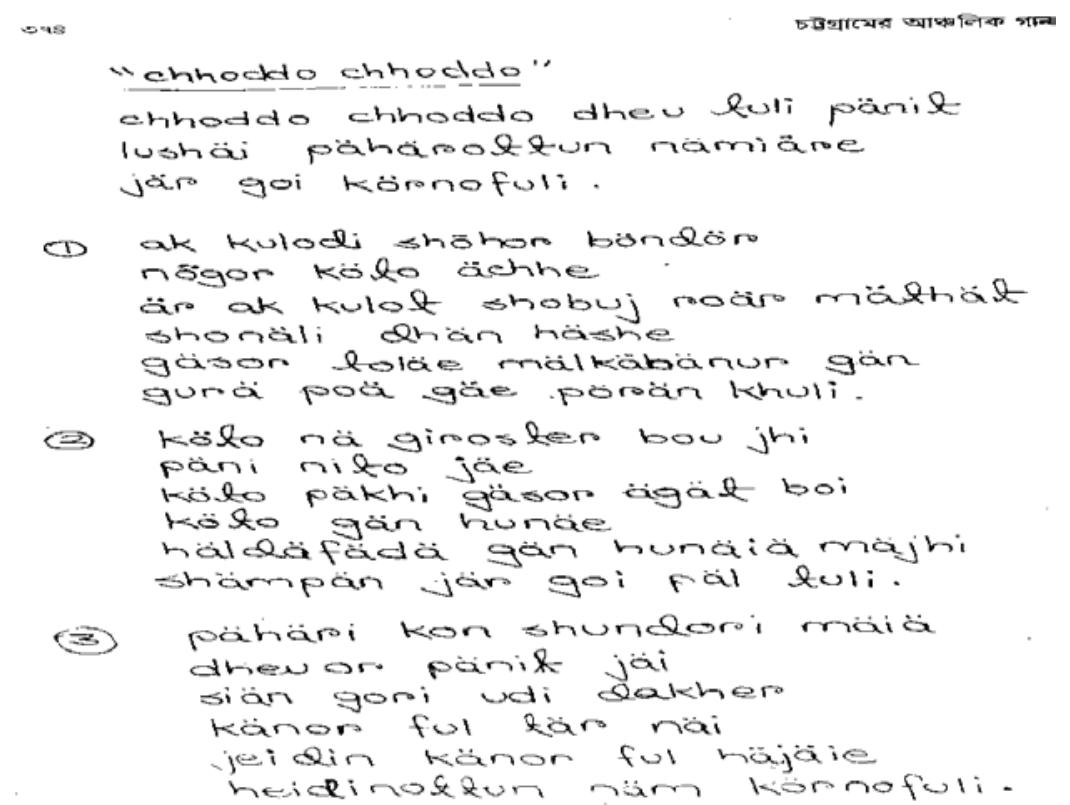

\subsection{Noticeable Differences between Chittagonian and Bangla}

Many linguists admit that there are many differences between Chittagonian and Bangla. In this regard BSC (2008, Preface) says: "In writing "Chottogramer Manush O Sankskriti", while visiting many villages, I have found some slangs that there have no similarity with Bangla. But they have much good taste and are meaningful.”

1.3.1. In vocabulary: A great number of Chittagonian words are found much different from Bangla. The people of other regions can hardly understand them. As a Chittagonian the researcher observes the difference noticeably. Here is a list of them from his observation:

\begin{tabular}{|c|c|c|}
\hline Chittagonian & Bangla & English \\
\hline $\begin{array}{l}\text { দৈজ্জা (doiffa)- } \\
\text { Urdu 'dorya' }\end{array}$ & সাগর sagor & Sea \\
\hline ফফর (foir) & পুকুর pukur & Pond \\
\hline ফন্না (fonna) & পড়ালেখা poralekha & Study \\
\hline বেইন্না (beinna) & সকাল sokal & Morning \\
\hline আযুইন্না (ajuinna) & সন্ধ্যা sondhya & Evening \\
\hline ওরোবারি (crobari) & শ্বশ্ররবাড়ি shashur bari & In-Law's house \\
\hline আমত্যা (amoțţa) & আবার abar & Again \\
\hline
\end{tabular}


Here is another list of words that shows completely different meaning, though they sound similar:

\begin{tabular}{|l|l|l|}
\hline \multicolumn{1}{|c|}{ Words } & \multicolumn{1}{|c|}{ Chittagonian } & \multicolumn{1}{c|}{ Bangla } \\
\hline Jhi (ঝি) & Daughter & $\begin{array}{l}\text { Both 'daughter' and } \\
\text { 'maid servant' }\end{array}$ \\
\hline Gom(গম) & good/well & corn \\
\hline Chata(ছাতা) & Dirt & an umbrella \\
\hline Bish(বিষ) & Pain & Poison \\
\hline
\end{tabular}

In sound system: There are noticeable differences between Chittagonian and Bangla sound systems. Some of them are as follows:

\begin{tabular}{|c|c|c|}
\hline & Bangla & Chittagonian \\
\hline 1. & Bilabial-Plosive (p/প) & labiodental fricative (f) \\
\hline 2. & $\begin{array}{l}\text { Bilabial voiced aspirated plosive } \\
\text { (bh/s) }\end{array}$ & $\begin{array}{l}\text { Bilabial unvoiced unaspitated plosive } \\
\text { (b): for example, 'bhala' of Bangla is } \\
\text { 'bala' in Chittagonian }\end{array}$ \\
\hline 3. & $\begin{array}{l}\text { unvoiced Velar aspirated plosive } \\
(\mathrm{kh} / \vartheta)\end{array}$ & Aspirated fricative \\
\hline 4. & unvoiced affricate $(\mathrm{c} / \overline{\mathrm{b}})$ & Unvoiced fricative (s) \\
\hline 5. & Voiced affricate (dz/জ) & Voiced fricative (z) \\
\hline 6. & Alveolar palatal plosive (ch/ছ) & Alveolar palatal fricative \\
\hline 7. & For the front vowels (e) and (æ) & $\begin{array}{l}\text { An irregular vowel }[\varepsilon] \text { which is lower } \\
\text { than [æ] and higher than }[\mathrm{e}] \text { ) }\end{array}$ \\
\hline
\end{tabular}

\subsubsection{In Grammar}

Chittagonian grammar is similar to that of Bangla, with significant variations in inflectional morphology (prefixes, suffixes, particles, etc.), and some variation in word order.

Like related languages of the eastern subcontinent, Chittagonian is a head-final language, with Subject-Object-Verb basic word order. Like Assamese (Ôxômiya) but unlike Bengali (Bangla), Chittagonian has preverbal negation. This means that the negative particle will precede the verb in Chittagonian, where the corresponding Bangla version would have a negative particle followingtheverb. (Wikipedia, 2010)

\begin{tabular}{|l|l||}
\hline \multicolumn{1}{|c|}{ Bangla } & \multicolumn{1}{|c|}{ Chittagonian } \\
\hline \hline Ami bhat khai na & I bát(rice) no hai (do not eat). \\
\hline She (He) tbh dekhche na & Ite tb(TV) no sa:r (watches). \\
\hline $\begin{array}{l}\text { She (she) shaikele chorchhe } \\
\text { na }\end{array}$ & $\begin{array}{l}\text { Iti(She) sairkélot(bicycle) no sorér(isn’t } \\
\text { riding). }\end{array}$ \\
\hline \hline
\end{tabular}




\subsection{Rationale}

For the above mentioned distinction in Chittagonian, different opinions are found regarding its being a language or a dialect among sociolinguists. Though it is considered a dialect of Bangla, it is far different from Bangla in many ways. It has some features which may claim it to be a separate language, such as it is not mutually intelligible with Bangla, even with other dialects of Bangladesh. So Kumar (2011) opines: “Although these languages (Sylheti, Chittagonian, and Chakma) are mutually intelligible with neighboring dialects of Bengali, a native speaker of Standard Bengali would hardly understand them.”

But as it does not have all necessary features to be a distinct language, such as having no official status, no writing symbols, etc., this study likes to present some necessary qualities which may contribute a lot to giving it the status of a separate language.

\subsection{Aim}

The researcher assumes that doing research on the above area will make a pathway to the consideration and recognition of the Chittagonian variety as a separate language that will enrich the number of languages of Bangladesh and the world as well. The questions that have been haunting him are as follows:

1. Is the Chittagonian variety a dialect or a language?

2. To what extent is it true to say that the Chittagonian variety is a dialect of Bangla?

3. If the Chittagonian variety is neither a language nor a dialect, what is it?

\subsection{Previous Literature and Chittagonian}

McArthur (1998, pp.139-142) in a longitudinal study has mentioned that the people of Scotland occupy a unique historical and cultural position in the English speaking world. They use the standard English in administration ,law, education, the media, all national institutions and by and large in their dealings with Anglophones elsewhere, but in their everyday life a majority of them mix 'the king's English' with 'the King's scots'(in an earlier age it was called). Again he mentioned that there was much discussion on the status of Scots and from that three broad points of view can be distinguished:

\section{A dialect of English}

For many, Scotland and elsewhere, Scots is obviously an English dialect. Though it is distinctive in its sound, spelling, syntax and vocabulary, it is 
not different enough structurally and lexically to be a language in its own right. Moreover, it has no official status, no significant presence in schools and administration, only a minor role in country's legal system.

\section{An independent language}

For many others, in Scotland And elsewhere, Scots is obviously a language, because:

(1) It has a highly distinctive sound system, grammar, and vocabulary, dating from the Anglian kingdom of Northumbria over a thousand years ago, long before either Scotland or England was a state.

(2) It has a varied and unbroken orthographic and literary tradition from the middle ages to the present day, including two medieval epic poems, ballads and love poetry, and writers of standing who have used Scots on its own or with English. These include John Barbour, 'Blind Harry', William Dunbar, Gavin Doglous, Sir David Lindsay, Allan Ramsay, Robert Burns, Sir Walter Scott, John Galt, Robert Louis Stevenson, Christopher Murray Grieve, Edwin Muir, James Leslie Mitchell, and Neil M. Gunn.

(3) It has dialects of its own ranging from the borders (with ancient links to Norhtern England) to the Northern Isles of Okney and Shetland (with ancient links to a variety of Old Norse called Norn).

(4) It is now recognized as language by the European Bureau of Lesser Used Languages, an agency of the European Union.

\section{A semi-language}

In 1985 A.J. Aitken, former editor of the Dictionery of the Scottish Tongue, stated, "If Scots is not now a full 'language' it is something more than a mere 'dialect'. A distinguished German scholar once called it a Halbsprache - a semi-language.”

Aitken's closing remark:

I was horrified to read that Scots has been called a Halbsprache in German: Halvsprak in Swedish is worse than 'half a language'! ...My German university friends assure me that if Low German is a language (and who denies that?), then Scots is bound to be a language. They can't understand why Scots shouldn't be allowed to be called a language, just like Luxemburgish, which is not taught in the schools of Luxembourg as a German dialect but a language. In my Geolinguistic Handbook, Scots will remain a language. 
Like Scots, Chittagonian has got many qualities which are strong evidence for the Chittagonian variety to be a language. Like Scots, it has a highly distinctive sound system, grammar, and vocabulary. It has dialects of its own. Finally and significantly, many are uncertain about where Chittagonian begins and ends in relation to conventional Bangla.

In this regard, the following opinion of Alam (2011) is notable:

If any dialect is the farthest from the Bangla language in pronunciations, sentence construction, and part-of-speech sequence, that is the dialect of my home district Chittagong. It is so remote from the standard Bangla language that once I did a little research as to find out whether the dialect came from altogether a different language or was a distorted colloquial form of Bangla. Let alone the uneducated people, the fact that most of the educated people of Chittagong can't speak Bangla (myself included) with the correct pronunciation, or do speak with a heavy accent owing to the reason that Chittagonian dialect has almost nothing similar to Bangla so far as the linguistic properties are concerned. But I found out that scholars such as Abdul Karim Shahitya Bisharad, Dr Enamul Haq and Abul Fazal (all from Chittagong) viewed Chittagonian as having stemmed from the standard Bangla. They couldn't tell me the derivation history of how the distortion to this degree took place, but they agreed that as Chittagong was bordering with the Arakan kindom of Burma, so a lot of interexchange between the Arakanian language and the Bangla spoken in Chittagong had taken place and the result was the Chittagonian dialect.

In another study of Wikipedia (2010), it is found that Chittagonian is an Indo-Aryan language spoken by the people of Chittagong in Southeastern Bangladesh throughout Chittagong Division but mainly in Chittagong and Cox's Bazar Districts and also in countries where many Chittagonians have migrated. Though it is closely related to Bangla, it is normally considered by linguists to be a separate language rather than a dialect of Bangla. It is estimated to have 14 million speakers spread in different countries. According to the status of Top 100 Languages by Population by Ethnologue, Chittagong ranked 69th of the world. It has got a long historical and ethnic background which can be observable in the following table. 


\begin{tabular}{|c|c|c|}
\hline Spoken in & Bangladesh, India, Myanmar & \\
\hline Region & \multicolumn{2}{|l|}{ Eastern South Asia } \\
\hline $\begin{array}{l}\text { Total } \\
\text { speakers }\end{array}$ & \multicolumn{2}{|l|}{14 million } \\
\hline $\begin{array}{l}\text { Language } \\
\text { family }\end{array}$ & \multirow{2}{*}{\multicolumn{2}{|c|}{$\begin{array}{l}\text { Indo-European } \\
\text { Indo-Iranian } \\
\text { Indo-Aryan } \\
\text { Easterr }\end{array}$}} \\
\hline & & \\
\hline & & $\underline{\text { Bengali-Assamese }}$ \\
\hline & & Chittagonian \\
\hline $\begin{array}{l}\text { Sister } \\
\text { languages }\end{array}$ & \multicolumn{2}{|c|}{$\begin{array}{l}\text { Sylheti (Silôți), Bengali (Bangla), Assamese } \\
\text { (Ôxômiya), Oriya, the Bihari languages and also less } \\
\text { directly all other Indo-Aryan languages such as } \\
\text { Hindi. }\end{array}$} \\
\hline ISO 639-1 & \multicolumn{2}{|r|}{ None } \\
\hline ISO 639-2 & \multicolumn{2}{|c|}{ Cit } \\
\hline ISO 639-3 & \multicolumn{2}{|r|}{ Ctg } \\
\hline
\end{tabular}

In an interview, Khan (2009) opines that the language that the middleclass poets of Calcutta have introduced is also a regional language. But it has got the status of a standard language for political and historical reasons. As a language many things are to be learnt from it. It does not mean that we have to look down upon the qualities of the languages of other regions. If we do so, it will be suicidal. If we can learn Greek and Italian as Madhusudhan learnt, we should learn the languages of Mymensingh and Barishal. He again maintains, "English is not a single language, but Multilanguage. So I have wanted to give a message that there is no single language named Bangla, but it is Multilanguage”. (Salimulla Khan, interviewed by Shams al Amin, New York/December 14, 2009/published on 18 August 2010/)

The observation of Wasif (2012) in a newspaper article is almost similar to the opinion of khan. He says that many think only the standard Bangla is the correct one, as dialects others are incomplete ones. This idea has no linguistic stand. The linguists do not justify the loftiness or the 
meanness of any language. They look into the varieties and the probabilities of a language. What is undermined as dialect or regional variety is the substance of a language. The oral language is the complete one. Most of the people speak that language.

So there is no chance to neglect Chittagonian, considering it as a dialect. Moniruzzaman (2007), on the contrary, opines that considering the phonology, morphology, and syntax of the Chittagong dialect; it is said that the variation of the language or the dialect surrounded by the sea and hills makes it nothing but one of the dialects of Bangla.

But referring to Mahbubul Haq's observation Alam (2012) argues that literature expert Abdul Karim in an article termed Chittagonian a separate and distinct language instead of a dialect, Muhammad Enamul Haq did so too.

Correspondingly, Ahmad (October, 2013) has said that like new Indian Aryan languages: Oriya, Assamese, Maithili, etc., Chittagonian is not any dialect of Bangla. Because according to his observation, the history of Chittagonian is older than that of Bangla. In his earlier work, he (September, 2013) argues that if intonation, word order, syntax, etc. of Caryapada are analysed, it will be proved that Chittagonian is older than Bangla. He has shown that some Carya words like 'বাকলত' 'দুআরত' ‘আইল’, ‘চীঅন’ (Carya 3), ‘উআস’' (Carya 7), ‘ঘলিলি’ (Carya 10), ‘উইত্তা’ (Carya 30), 'উজু' (Carya 32), 'টালত' (Carya 33) and others are exactly found in Chittagonian. Moreover 'চানসুজ্জ' of Chittagonian is derived from Carya verse ‘চান্দ সুজ্জ দুই চাকা সিটি সংহার পুলিন্দা’(Caray 14). He again observes that the preverbal negative marker of Chittagonian is found in many verses of Caryapada, e.g. 'দুলি দুহি পীঢ়া ধরণ ন জাই (Carya 2). And 'জে জে আইলা তে তে গেলা।'(Carya 7) seems to be the old form of present Chittagonian 'জে জে আইল তে তে গেইল।'. Thus he observes that Chittagonian is apparently older than Bangla and from 650 to 1200 C.E. Chittagonian was seemingly like Caryapada. He further opines that before the arrival of foreign traders in a great number, the Chittagonian would speak in the Prakrit language, instead of Bangla.

In his later studies, he rightly says that for the strong theoretical foundation of the Bengali language, Muhammad Shahidullah, Sunitikumar Chattopadhyay, Sukumar Sen, Professor Muhammad Abdul Hai and others have worked hard, whereas in the case of Chittagonian 
such attempts by any scholar were absent. He acknowledges that Moniruzzaman paved the way for Chittagonian to be studied, but his study was based on Northern-Chittagong in which root form of Chittagonian was absent. He clarifies that in the Northern and Metro area, Chittagonian has been corrupted greatly by other languages. In this regard in his earlier studies (September, 2013), he opines that Arakani effect is a little more on the southern regions and on the Northern region there is the influence of the regional languages of Tripura and Noakhali. But excluding the speeches of riparian people of Feni and Napha rivers, unity is observed in the speeches of the riparian people of Karnaphuli, Shankha, Matamuhuri and Bankkhali rivers with very slim differences. So as a model for a standard or universally accepted forms of Chittagonian, the speeches of Chittagong city, Hatahajari, Raujan, Boalkhali, Patiya, Chandanaish, Anowara, Banskhali, Satkaniya, Lohagara, Chakariya and so, can be taken.

In his further observation, he says though Moniruzzaman has been staying in Chittagong for a long time, he is from Norshingdi. In this regard, his earlier observation is that those who are deep-rooted Chittagonian can study the Chittagonian language better. It is difficult for the speakers of other regions.

Regarding dialects as well as Chittagonian, Morshed (2007) states that variation is found in the dialects of the different regions in Bangladesh. These occurred mainly in sounds, morphemes and sentences. But in a comparative study, the variation in morphemes is seen a lot. In sound system of the dialects, though the vowels and consonants of standard Bangla are used, there is distinction of this in some contexts. This is very much observed in the Chittagong dialect. He again says that among the dialects of Bangladesh, variations are seen most in the dialects of Rangpur and Chittagong. There is historical reason behind the fact. Before the arrival of the British, a relation of this region in the fields of trade and immigration had been established with non-Bangla speaking areas. And as a result, many foreign linguistic elements and morphemes penetrated here. The variations in the Rangpur dialects resulted from this. The Chittagong dialect, for the same historical background has been influenced by the Arakanese Mogh and Arab-Portuguese traders. (xxii/ xxiii, Introduction) 
In another study through internet search $\sim$ Slsmhu (2011) found that Rohingya, a language spoken by the Rohingya Muslim people of Arakan (Rakhine), Burma (Myanmar), is linguistically similar to the Chittagonian language spoken in the neighboring south-eastern Chittagong Division of Bangladesh. It also has a large number of Urdu, Persian, Hindi, Arabic, Burmese and English loanwords.

Rohingyalish is the modern writing system of the Rohingya people. The word Rohingyalish is derived from the two words Rohingya and English due to the fact that it uses mainly Roman script which is completely different from that of the previously used scripts such as Arabic, Urdu, Hanifi-Script and Burmese.

So if Rohingya which is almost similar to the Chittagonian variety can be a language, why can it (Chittagonian) not be a language? Then Chittagonian is a dialect of Rohingya or Rohingya is a dialect of Chittagonian.

\subsection{Methodology}

A researcher gathers various kinds of data from various sources and among the data quantitative or qualitative data are prominent. Quantitative data are objective facts capable of measurement and eventuating into statistical concepts. On the other hand, Qualitative data are subjective, impressionistic information, which are gathered by the use of open-end interviews, observation and case studies. As the research is a kind of desk work, based on the nature and the necessity of the selected research area, the researcher has followed Qualitative method in this particular research project. In carrying out the research, he had the privilege to gather information by random sampling. This was on the basis of collecting newspaper articles, books, and web sources, etc. In doing so, the researcher of this paper has taken enough liberty in translating some information of some newspaper articles written in Bangla.

\subsection{Discussion and Findings}

In the literature on this paper, the arguments found in favour of the status of Chittagonian as a language are stronger than those found against the status. So considering literature and information found in this 
study, it can be said that Chittagonian variety can be termed a distinct language for the following reasons:

1) It is not mutually intelligible with Bangla. The following opinion of Syal and Jindal (2008) is remarkable here:

It is sometimes very difficult to say whether A and B are different languages or just different dialects of the same language. The partial solution to this problem is provided by the concept of mutual intelligibility. If two speakers are mutually intelligible, they are using the same language even if they are using different dialects. If they are not intelligible to each other, they are using different languages.

2) According to the information of Wikipedia, though Chittagonian is closely related to Bangla, it is normally considered by linguists to be a separate language rather than a dialect of Bangla.

3) According to the same source, in the status of Top 100 Languages by Population by Ethnologue, Chittagong ranked 69th of the world.

4) It has many other sister languages beside Bangla (Sylheti (Silôți), Assamese (Ôxômiya), Oriya, the Bihari languages and also less directly all other Indo-Aryan languages such as Hindi.). (Source: Wikipedia).

5) It has highly a distinctive sound system and distinctive grammar, and vocabulary dating from the Aryan history.

6) It has its own rich oral literature. Some literature written in Bangla letters is also found. Even in some Bangla literature of the poets from Chittagong background, some words of Chittagonian variety are found.

7) It has dialects of its own ranging from the borders. In this case, the opinion of Abdul Karim Shahitya Bisharad is supportive: the Chittagonian language has lost its purity when it crossed Cox's Bazar. It is because of the influence of Rosanga.

However, from some information in the literature, Chittagonian variety cannot be termed a language; rather it should be termed as a dialect of Bangla for the following reasons:

a) It has no official status and is not taught at any academic level.

b) It has no writing symbols.

c) Its literature is not rich enough for the status of a language.

d) Its vocabulary and grammar are similar to those of Bangla, though there are certain differences. 


\subsection{Counter-arguments}

Regarding the official status, the study observes that it is not a precondition for consideration of a variety to be a language. There are many languages in the world which do not have any official status. In this case, the opinion of Spolsky (1998, p. 30) is noteworthy:

"A language, it has been remarked, is a dialect with a flag, or even better, with an army."

Regarding writing symbols we can say that they can be developed following the symbols of any other language, as we know that Urdu writing symbols have been developed following the symbols of the Arabic language. Regarding literature, it can be said that they can be developed in course of time.

\subsection{Limitations}

Though some information is found in favour of the status of Chittagoian as a language, the study has many limitations in presenting the necessary arguments, so some more studies are needed in support of the claim of the researcher. But the present researcher objects to the existing idea that Chittagonian is a dialect, a substandard and distorted form of Bangla. In this respect, his observation is to regard it at least as something more than a dialect, i.e. a semi-language.

\subsection{Observations and Recommendations}

1) If Rohingya which is almost Chittagonian can have writing symbols, why cannot the writing symbols of Chittagonian be developed? So the study recommends developing the writing symbols. It can be done using letters both from Bangla and English, if necessary even from other languages. Because we all know that Urdu writing symbols have been developed using the writing symbols of Arabic. We also came to know from literature on this paper that Rohingya got its writing symbols using Roman scripts.

2) Literature in Chittagonian can be developed using Bengali writing symbols until the separate writing symbols for Chittagonian are developed. This practice is going on in some Chittagonian daily newspapers like The Daily Azadi. 


\subsection{Conclusion}

In conclusion, it can be said that though Chittagonian is considered a dialect of Bangla, it is far different from Bangla in many respects, as we have come to know in this study. For this distinction, it has got most of the qualities of a distinct language. Though still some more studies are to be done for the status of Chittagonian, it is not difficult for it to be developed into a language. The researcher hopes his findings will no doubt encourage the language teachers and the linguists to contribute to the development of Chittagonian variety as a language.

\section{References}

Ahmad, K. (2013, September). Chattagrami Bhasha ki ekti shotontro Moulik Bhasha? (Is Chittagonian a Distinct Original Language?) [Electronic version]. The Daily Azadi, the Anniversary version.

Ahmad, K. (2013, October). Chattagrami Bhasha Niye Dr. Moniruzzamaner Gobeshona (Moniruzzaman's Research on Chittagonian) [Electronic version]. The Daily Azadi.

Alam, M. U. (2011). Language And Dialect: An Emerging Cultural Conflict. Retrieved 19 January, 2011, from www.daily-sun.com/?view...ds.../

Alam, S. (November, 2012). Mahbubul Haq er Chattagram Onnesha (Mahbubul Haq's Inquisitiveness for Chittagonian) [Electronic version]. The Daily Azadi.

BSC. N. I. (2011, April). Shahitya O Chottogramer Ancholik Bhasha (Literature and Chittagonian Local Dialect) [Electronic version]. The Daily Azadi, 5.

BSC, N. I. (2008). Chattagramer Buli O Gali (Slang and Proverbs of Chittagong). Chittagong: Amader Pathshala (Our Library)

Crystal, D. (2003). A Dictionary of Linguistics and Phonetics (5 ${ }^{\text {th }}$ ed.). Oxford: Blackwell Publishing.

Chittagonian language. (2011). Retrieved December 4, 2011, from http://www.enotes.com/topic/Chittagonian_language/

Ghosh, K. (1998). Chattagramer Ancholik Gan (Local Songs of Chittagong). Dhaka: Bangal Academy.

Hasan, M. (2010). Adorn Chattogrami Banglar Avidhan- A Dictionary of Chittagonian Bangla( $1^{\text {st }}$ ed.). Chittagong: Adorn Publication.

Kashem, A. M. (2011, June). Chatga Bhashar Itihash Onnesha (Looking for the history of Chittagonian Language) [Electronic version]. The Daily Azadi, 5. 
Kumar, R. S. (2011). Bengali - One language, Multiple Variations. New Delhi. Retrieved 5 December, 2011, from http://www.translationdirectory.com/article1069.htm/

Moniruzzaman. (2007). Language and Literature (Cultural Survey of Bangladesh Series-6). Dialect of Chittagong (pp.236-245). Z. I. Ali. Trans. In Morshed, A.K.M. (Ed.). Dhaka: Asiatic Society of Bangladesh.

McArthur, T. (1998). The English Languages. Cambridge: Cambridge University Press.

Richard, J. C., et al (1993). Longman Dictionary of Language Teaching and Applied Linguistics (p.107). ( $3^{\text {rd }}$ ed.). UK: Longman.

Slsmhu (2011). Interactive Language. Retrieved December 5, 2011, from http://www.interactivelanguage.net/bengali-language/rohingya- language-isnot-a-dialect-of- bengali-language-yes-or-no/

Syal, P. \& Jindal, D.V. (2008). An Introduction to Linguistics: Language, Grammar and Semantics ( $2^{\text {nd }}$ ed.). The Study of Language Variation (P.58). New Delhi: Prentice Hall of India Private Limited.

Spolsky, B. (1998). Sociolinguistics. Oxford: Oxford University Press.

Wardhaugh, R. (2006). An introduction to Sociolinguistics (5 ${ }^{\text {th }}$ ed.).Oxford: Blackwell Publishing.

Wikipedia. ( 2010) Chittagonian Language.. Retrieved ctober7, 2010, from http://en.wikipedia.org/wiki/Chittagonian_language\#Nasal_vowels/

Wasif, F. (2012). Bangla:Dorbari, Ejmali Na Dhakai (Bangla: Courtly, Gereral or Dhakai) Prothom Alo (Electronic version), 23-02. 